\title{
Arts de guérir: psychologie médicale, médecine par- allèle et écriture chez Hervé Guibert
}

\section{Stéphane Spoiden}

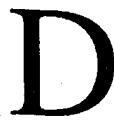

ans $A$ l'ami qui ne m'a pas sauvé la vie, Hervé Guibert raconte qu'il avait un jour demandé à Muzil, qui n'est autre que Michel Foucault, quelle était sa position sur la question de la vérité à propos de la maladie fatale dans le rapport entre médecin et patient. Foucault répond: "'Le médecin ne dit pas abruptement la vérité au patient, mais il lui offre les moyens et la liberté, dans un discours diffus, de l'appréhender par lui-même, lui permettant aussi de n'en rien savoir si au fond de lui il préfère cette seconde solution"' (A l'ami 33). Bien que Guibert développe plus tard avec le docteur Chandi ce qu'il appelle "une relation bienfaisante" digne des attentes de Foucault (Le Protocole compassionnel 81), force est de constater à la lecture de l'œuvre du sida de Guibert que le niveau de psychologie généralement manifesté par l'institution médicale est bien en deçà de l'optimiste remarque du philosophe. D'ailleurs, Foucault ne se leurrait nullement quant au caractère déshumanisant de la pratique médicale. Guibert se remémore encore ce qu'en disait Foucault à la suite d'une visite médicale qu'il eut à effectuer en 1983:

Muzil passa une matinée a l'hôpital pour faire des examens, il me raconta à quel point le corps, il l'avait oublié, lancé dans les circuits médicaux, perd toute identité, ne reste plus qu'un paquet de chair involontaire, brinquebalé par-ci, par-là, à peine un matricule, un nom passé dans la moulinette administrative, exsangue de son histoire et de sa dignité. (A l'ami 32) 
Ces récriminations lancées à l'encontre du corps médical ne sont pas rares puisque la médecine moderne depuis qu'elle a vu le jour durant le siècle passé n'a cessé d'accuser une carence en matière psychologique. Au dix-neuvième siècle, le succès scientifique de l'école anatomo-clinique, le passage de l'anatomie générale à l'anatomie microscopique et le développement des connaissances en physiopathologie provoquent une transformation profonde de la pratique médicale. ${ }^{1}$ La médecine devient l'étude des dysfonctions et porte peu d'intérêt aux entités. Le médecin ne traite plus un être humain mais des organes, des fonctions anormales ou dans le meilleur des cas un porteur de maladies. Le médecin devient un technicien, un technocrate avec honoraires qui relate la maladie comme l'expression de lésions d'organes ou comme trouble de fonctionnement d'une mécanique (Médecine 671-72). Ce regard médical déshumanisant et cette indifférence vis-à-vis du vécu et de l'angoisse du patient engendrent depuis la fin du dix-neuvième siècle et régulièrement depuis lors, une perte de confiance en la médecine scientifique et ouvrent la porte aux médecines parallèles qui, de leur côté, séduisent en misant sur les carences du monde médical scientifique. ${ }^{2}$

A partir de ce constat, je me propose, premièrement, de faire ressortir des romans et du journal d'hospitalisation d'Hervé Guibert le parcours typique et observable du malade qui perd peu à peu confiance en la médecine scientifique moderne et qui est tenté, malgré une lucidité hors du commun et une rationalité certaine, de chercher secours et espoir dans la médecine parallèle ou nonmédecine; deuxièmement, de déterminer ce que la médecine parallèle, notamment par l'entremise d'un guérisseur au Maroc, offre comme alternative; et finalement, d'analyser brièvement les stratégies déployées par Guibert dans les relations de pouvoir qui prennent place dans le rapport corps médical - patient.

Les exemples d'insuffisance psychologique du monde médical abondent dans les récits de Guibert: la lourdeur et l'intransigeance bureaucratique, les manquements à la déontologie et à l'éthique les plus élémentaires, la pauvre qualité des soins prodigués sont des lieux communs. Avant même qu'il n'ait à se soumettre aux examens requis par le traitement du sida, Guibert avait déjà eu un "avant-goût" de la condition de patient dans sa relation au monde médical. D'une part, les angoisses de son hypocondrie l'avaient déjà amené à endurer les affres d'une urographie opérée dans les pires conditions d'humiliation et d'horreur: ${ }^{3}$ [U]ne épreuve terrible, humilié, couché nu plus d'une heure, alors qu'on ne m'avait pas prévenu de la durée de cet examen, sur une table de métal glacée, sous une verrière où pouvaient me voir des ouvriers qui travaillaient sur un toit, impuissant à appeler quiconque car on $m$ 'avait oublié, une aiguille épaisse plantée dans la veine du bras diffusant dans mon sang un liquide violacé qui le chauffait à mort.... (A l'ami 41)

D'autre part, Guibert voit à travers l'hospitalisation de Foucault peu avant sa 
mort, dont il relate quelques épisodes particulièrement ignobles, la préfiguration de son devenir:

A l'intérieur de la chambre de réanimation c'était un bordel incroyable, un nègre houspillait la soeur de Muzil ... Il dit qu'on n'était pas dans une bibliothèque, il attrapa les deux livres de Muzil ... qui sortaient tout frais de l'imprimerie, et décréta que même ça on n'en voulait pas ici.... (103)

Guibert, en accompagnant son grand ami dans ses derniers moments, partage en quelque sorte le triste sort de nombreux sidéens qui voient partir leur partenaire-image projetée de leur futur proche: “[C]'était désormais une certitude qu'en plus de l'amitié nous étions liés par un sort thanatologique commun" (102).

Mais pour Guibert, le véritable "premier contact" avec l'institution hospitalière en tant que sidéen constitue également un des épisodes les plus marquants! Guibert est envoyé à l'hôpital Claude-Bernard, très anxieux, afin d'y subir ses premiers tests en vue de l'obtention du médicament AZT. Il se rend compte, sans qu'on l'ait prévenu, que l'hôpital devenu insalubre est désaffectéet les différents services transférés dans de nouveaux locaux-, excepté le "bâtiment exclusivement affecté aux malades du sida et en fonctionnement à l'intérieur de l'hôpital mort" (A l'ami 51). L'incursion de Guibert dans le labyrinthe hospitalier confirme cette isolation suspecte. Un parcours mal fléché parmi des bâtiments lugubres et aux noms peu réjouissants fait office de "service d'accueil," lui aussi transféré. De "Maladies infectieuses," Guibert passe par "Epidémiologie africaine" pour enfin aboutir au "Pavillon des maladies mortelles" (53). Arrivé à destination, Guibert doit alors endurer le véritable service d'accueil, celui en blouse et chaussures blanches. Le personnel n'a que faire des moindres principes de discrétion et de bienveillance qu'un minimum de compréhension de l'état psychologique des patients visiteurs prescrirait. Guibert expérimentera encore une telle pratique dénuée de toute considération déontologique ou même simplement humaine lors de son ultime hospitalisation lorsqu'une infirmière lui lancera malicieusement: "Qu'est-ce qui est préférable, une injection dans l'œil, moi je sais que je ne supporterais pas, ou devenir complètement aveugle ?"' (Cytomégalovirus 33).

Dans Le Protocole compassionnel, son deuxième livre sur le sida et suite de $A$ l'ami, Guibert relate avec détails les deux fibroscopies qu'il eut à supporter, la deuxième, un modèle de soins, servant de contrepoint comparatif à la première qui, celle-ci, relève de la torture. ${ }^{4}$ Guibert, ayant commis "l'erreur" de se présenter sans dossier ni lettre de recommandation, eut droit au régime "ordinaire" prodigué par l'assistant débutant et terrorisé, et à l'inattention cynique du docteur responsable. Guibert conclut après coup: "Pour le docteur Domer," qu'il caractérise au passage de sadique nazi, "je n'étais qu'un petit pédé infecté de plus, qui allait de toute façon crever, et qui lui faisait perdre son temps" (58). La 
terreur que suscite l'examen médical est encore parfaitement exprimée dans la description d'une salle d'attente où les patients horrifiés hésitent malgré leur délabrement physique, ou plutôt en raison de leur état désespéré, à subir une opération insupportable:

Nous étions trois à attendre l'examen, assis en rang d'oignon près de cette porte derrière laquelle il se faisait, essayant de comprendre les allées et venues, guettant les bruits qui nous parvenaient et cherchant à apercevoir dès que cette porte s'entrouvrait une partie de l'appareillage qui allait nous torturer. (40)

Ce type d'examen standardisé, impersonnel et peu compréhensible pour le patient est d'autant plus déplaisant qu'il reproduit le sentiment d'invasion que suscite le sida lui-même. Les nombreuses interventions que subit Guibert comportent immanquablement un appareillage qui s'insère dans le corps et suit douloureusement ses méandres à l'image du virus VIH qui s'insinue dans le sang. Guibert décidera de mettre fin à ces pratiques trop réminiscentes de sa maladie au risque de voir sa piètre santé se dégrader davantage: "Je refuse les endoscopies: fibroscopie, coloscopie, lavage alvéolaire, tuyaux dans la gorge, dans le cul, dans les poumons, j'ai déjà donné" (Le Protocole compassionnel 28). ${ }^{7}$ En outre, la métaphore de pénétration et d'envahissement comporte une signification psychologique personnelle pour l'auteur que seul le docteur Chandi ménage. Guibert nous informe dès le début de son premier roman que depuis tout petit il refuse le bâtonnet de bois couramment utilisé pour l'auscultation de la gorge, qui pour lui est "truffé d'échardes mentales" (A l'ami 19). Au viol du corps, s'ajoute également cette sensation de vol par les quantités astronomiques de sang qu'on lui prélève régulièrement. Guibert finira par négocier avec le personnel médical vampirique le nombre de tubes à lui soutirer pour les analyses, "Tout est marchandage dans la vie" dit-il (Le Protocole compassionnel 227). Les ponctions lombaires, ridiculement inutiles, opérées sur Michel Foucault à l'agonie quelque six ans plus tôt,-_autre signe prémonitoire-avaient préparé Guibert à affronter les prélèvements sanguins et sanguinaires.

La dépossession du corps et la perte de contrôle et de liberté sur la maladie sont encore accentuées par l'utilisation d'un jargon par le corps médicalqui aliène le patient-et surtout par l'incapacité générale des médecins à traduire les diagnostics en termes simples. Par la force des choses, Guibert finit par quelque peu s'en accommoder: "J'aimerais manier parfaitement le jargon des médecins, c'est comme un truc codé, ça me donne l'illusion vis-à-vis d'eux de ne pas être le gosse devant lequel on parle anglais pour des histoires de cul" (Le Protocole compassionnel 105). Le manque de cure et la difficulté à traiter un syndrome protéiforme amplifient encore ce sentiment de malaise entre soigné et soignant. ${ }^{6}$ Le degré élevé de confiance exigé dans toute relation entre médecin et patient s'estompe considérablement quand la médecine scientifique est incapable 
de rendre compte de la biologie et de la pathologie de la maladie. ${ }^{7}$ Dans le cas d'une maladie incurable comme le sida, la thérapeutique prodiguée par le milieu médical est souvent complexe, incompréhensible et incertaine. Elle affaiblit ou annihile la capacité du malade à se soigner et finalement l'"exproprie" de sa santé et de son corps. Quand Guibert confie à Bill, manager d'un grand laboratoire pharmaceutique, qu'il a le sida, il sent qu'il perd "toute liberté et tout contrôle sur sa maladie" (A l'ami 15). Son périple est parsemé de diagnostics et de prescriptions contradictoires. Les avis divergent sur les causes d'aggravation de son état quand Guibert en pleine dépression voit simultanément quatre médecins au début du Protocole compassionnel. C'est sous l'insistance de son entourage et non du corps médical qu'il se résout enfin à prendre du Prozac, un antidépresseur, que tous ses amis lui conseillent. La méfiance que manifeste Guibert à l'encontre de la pharmacopée s'en trouvera accrue par les diverses tergiversations des médecins et les déclarations alarmistes sur les effets néfastes de nouveaux traitements non testés. Les doses d'AZT et de DDI, les deux principaux médicaments que Guibert a utilisés, font l'objet de désaccords entre médecins et de compromis pour le moins suspects mais néanmoins révélateurs d'un aveu d'ignorance:

Que vous débutiez maintenant ou plus tard, que vous arrêtiez demain et repreniez après-demain n'a aucune sorte d'importance, parce qu'on ne sait rien à ce sujet. Ni quand on doit commencer le traitement, ni à quelles doses. Celui qui vous dira le contraire vous mentira. Votre médecin en France vous prescrit douze gélules, moi six, alors coupons la poire en deux, disons huit par jour. Le docteur Chandi qualifiera ensuite ces propos de dangereux. (A l'ami 229)

L'AZT aux effets secondaires nocifs s'avère insupportable pour Guibert qui l'abandonne au profit du DDI. Ce nouveau pharmakon, remède ou poison selon la dose, suscite, comme pour le Prozac, cette même attitude chargée d'hésitations et d'ambiguïté chez Guibert; une réticence exacerbée par la double inscription de la mort dans ce médicament qui a le pouvoir de tuer ou de sauver, et obtenu suite au décès de son premier destinataire. Emily Apter note à propos que Guibert est constamment hanté par "le spectre de [ce] danseur mort" (89). En effet le DDI, promesse de vie et ombre de mort, procure à Guibert un sursis revitalisant qui lui permet de reprendre entre autres ses activités littéraires: "C'est mon âme que je dissèque à chaque jour de labeur qui m'est offert par le DDI du danseur mort" (Le Protocole compassionnel 80). Ce sursaut d'espoir n'empêche pas Guibert de prendre peu à peu ses distances vis-à-vis des instances médicales. Après une période de familiarisation à sa dégradation physique, quelque peu ralentie par le DDI, Guibert se désintéresse des diagnostics, "récupère" son libre-arbitre, se surinforme sur la maladie et cherche d'autres possibilités thérapeutiques.

Avant d'être tenté par les médecines alternatives, Guibert mise sur ses 
relations dans les milieux médicaux afin d'obtenir le dernier médicament prometteur, de participer aux dernières expérimentations ou d'être l'heureux bénéficiaire de l'intangible vaccin curatif. Le monde pharmaceutique apparaît complètement inféodé à la loi du profit et dépourvu de psychologie dans ses pratiques expérimentales telles que le principe du double aveugle, justifiable sans doute pour la recherche mais particulièrement sinistre pour les participants. Même si Guibert bénéficie de quelques manipulations de protocoles, il n'en reste pas moins, et pour cause, que le monde médico-pharmaceutique ne brille pas par ses qualités humaines. Guibert dénonce également les nombreux personnages qui utilisent le sida comme "espoir de positionnement et de reconnaissance publique" (A l'ami 132). A l'ami qui ne m'a pas sauvé la vie-le titre ne pourrait être plus explicite-est un réquisitoire violent contre Bill, son supposé ami qui lui fait croire de manière éhonté à un remède bidon. Le microcosme que compose l'entourage de Bill (le docteur Chandi, Guibert et quelques-uns de ses amis) représente une image réduite, une synecdoque des agissements infâmes de l'industrie pharmaceutique. A travers ce sinistre individu épris de fantasmes de contrôle et de toute puissance, Guibert s'en prend à la domination qu'exerce l'industrie pharmaceutique sur le monde médical et par conséquent sur les consommateurs.

A défaut de renoncer totalement aux soins de la médecine scientifique, Guibert "s'ouvre" néanmoins à d'autres alternatives: avec circonspection et sans grand espoir à l'acupuncture et à l'homéopathie (médecines officialisées en France), en dépit de l'enthousiasme de son entourage; avec amusement aux suggestions de lecteurs, pas toujours bienveillants, qui proposent crudothérapie, injections mystérieuses et onéreuses d'hormones animales. ${ }^{8}$ La croyance religieuse le tente un instant lors d'un voyage au Portugal où il se résout à déposer des ex-voto dans une église célèbre pour ses miracles (A l'ami 207-11). L'offrande finira dans une poubelle de Lisbonne. Mais la tentation et l'espoirle dernier espoir! - se fera plus intense quand Guibert au bout du rouleau, en train de mourir, se rendra au Maroc chez un guérisseur aux pouvoirs exceptionnels (Le Protocole compassionnel 174-217). Celui-ci se présente comme l'image inversée de la médecine scientifique. Il noue d'emblée avec Guibert un rapport humain réel: il l'assure de son support et de son aide totale car il le considère désormais comme un fils. Guibert remarque, rassuré mais non sans ironie, que ce guérisseur n'a pas le profil typique du vieux gourou ou l'éclat du charlatan mais bien des allures de "chanteur d'opérette" (194). De fait, ce personnage cherche visiblement à se distancier au niveau de sa pratique de toute caractérisation charlatanesque: il promet la discretion, ne veut pas entendre parler d'argent et assume une certaine incompréhension-feinte ou réelle-de ses dons. A l'inverse du colloque médical classique qui exige confiance et soumission de la part du patient et autorité de la part du médecin, le guérisseur offre une relation égalitaire 
(tutoiement d'entrée) en avouant avant d'"ausculter" Guibert qu'il ne connaît pas le mal dont il souffre, mais qu'ils vont tenter de l'anéantir ensemble. Le guérisseur ne minimise pas non plus la maladie: après quelques minutes de magnétisme, il avoue n'avoir jamais vu un mal semblable, qu'il caractérise de cancer inversé. Bien que ses commentaires n'établissent qu'une collection de clichés sur le virus VIH comme "En tout cas il n'aime pas l'oxygène, ce virus" (197), ceuxci contribuent néanmoins à "l'authenticité" de sa pratique. Après une intervention anormalement longue d'une heure, si l'on admet qu'il guérit une méningite en cinq minutes, il offre une explication simple de la maladie et une thérapeutique claire et définitive qui tranche avec les tergiversations et procédures incertaines de la médecine scientifique. Tout en réfutant qu'il fait de la simple manipulation psychologique: "Et surtout pas de méthode Coue, hein!" s'exclame-t-il (198), ${ }^{11}$ le guérisseur propose à Guibert de prendre du magnésium et de boire un verre d'eau tous les jours à heure déterminée de façon à ce qu'il puisse se concentrer sur son patient au même moment. Ensuite, il explique que le virus s'autodétruira "un peu comme un scorpion qui se pique avec sa queue" (200). Une explication limpide de la maladie et de sa rémission, une promesse de guérison maintes fois réitérées semblent convaincre momentanément un malade en proie au désespoir.

Parallèlement à cette escapade dans le monde de la non-médecine qui sera évidemment sans lendemains, Guibert essaie néanmoins de modifier cette relation de contrôle de la maladie et du corps du patient imposée par l'instance de pouvoir que forme le monde médical et hospitalier. Guibert démontre que le pouvoir de l'institution médicale se définit à travers la dynamique qui se crée entre les opérations régulatoires, les modes disciplinaires imposés par les médecins et les formes de résistance opposées par le patient. ${ }^{10}$ Outre les amitiés de longue date qu'il entretient avec certains médecins, il cherche à établir une relation plus personnalisée avec une jeune femme médecin, Claudette Dumouchel, qui se montre d'emblée réticente aux "avances" de Guibert. Cette relation "amoureuse" à caractère compensatoire, qu'elle soit réelle ou fictionnalisée, dénote à souhait l'ampleur du déficit relationnel entre médecins et patients que ressent Guibert. Celui-ci déploie une stratégie de séduction et développe une relation fantasmatique qui ne visent en fait qu'à relâcher la tension et le contrôle qu'exerce sur lui l'institution médicale. Au bout de plusieurs mois, quand Claudette Dumouchel est enfin moins revêche à ses approches, Guibert raconte, à la doctoresse ennuyée, un cauchemar qu'il a fait et dans lequel elle l'abandonne en disant: “'Monsieur Guibert il n'y a pas que vous au monde!", Guibert répliquant, "'Mademoiselle Dumouchel, je ne sais pas si cet abandon ne relève pas de ce qu'on appelle une faute professionnelle"' (Le Protocole compassionnel 221).

"Le médecin est impuissant, sinon psychologiquement, à tendre le leurre que la maladie peut être reculée, sinon vaincue" (Le Protocole compassionnel 166). L'accusation est pratiquement sans appel: sinon psychologique- 
ment..., mais à l'exception du docteur Chandi, l'entourage médical de Guibert se montre précisément incapable de remplir cette ultime et unique tâche face à l'implacable maladie. Emily Apter suggère que la distance médicale entre patient et médecin imposée par l'éthique ne peut être maintenue avec ce type nouveau de maladie qu'est le sida et qu'il est nécessaire de réinventer la relation (95). C'est indéniable mais ce serait ignorer la plus grande partie de l'histoire des maladies que d'affirmer que le sidéen au seuil de la mort et sans espoir de guérison soit dans une situation inédite, et que le sida soit la première maladie à remettre radicalement en question la relation entre soignant et soigné. Le geste symbolique d'unité entre médecin et patient observé par Apter dans le projet de Guibert de filmer une intervention chirurgicale, qui inclut immanquablement le docteur, révèle dans le sens photographique non seulement le souhait de voir se créer une nouvelle relation en regard du sida, mais aussi de reconsidérer l'état lamentable de la pratique médicale aux niveaux psychologique et humain, quelle que soit la maladie. La problématique relationnelle soulevée par le sida parachève la remise en question généralisée du colloque médical, observée depuis de nombreuses années déjà parmi des patients qui requièrent un usage intensif de soins hospitaliers comme les hémophiles et les dialysés ou dans les revendications des associations contre le cancer. ${ }^{11} \mathrm{Et}$ bien avant l'émergence sociale de cette contestation ou la parution de réquisitoires violents contre la médecine (Némésis médicale de Ivan Illich), le champ littéraire, avec notamment Le Pavillon des cancéreux d'Alexandre Soljenitsyne, avait déjà dénoncé l'inacceptable rigidité de la stricte binarité du paradigme pasteurien. Cette "grande bataille napoléonienne", pour reprendre l'expression d'André Glucksmann (116), fondée d'une part sur la soumission et l'ignorance du patient, et d'autre part sur une application de techniques positives de savoir confortées par quelques victoires retentissantes au vingtième siècle (tuberculose, poliomyélite...) ne peut résister au nouveau défi que constituent le sida et la résurgence de maladies infectieuses que l'on croyait à jamais vaincues. ${ }^{12}$ Le geste de Guibert ainsi que son comportement général visà-vis de l'institution médicale est un appel à une médecine participative transparente où le soigné s'automédicalise et s'informe et où le soignant, en même temps que d'admettre sa relative ignorance ou incompétence, doit reconsidérer la teneur fondamentale du regard médical. La pratique médicale se doit désormais de dépasser le champ délimité de son investigation-le corps individuel et ses dysfonctions - et tenir compte de la charge psychologique individuelle et collective qu'il incarne. Herzlich et Pierret en 1984, avant que tout commentaire sur l'ébranlement définitif du paradigme pasteurien causé par le sida ne soit possible, concluent déjà que:

C'était vrai dans le passé, face à un savoir médical incertain; mais même aujourd'hui où elle est forte de la légitimité de la science, la médecine s'inscrit dans le social : le médecin est dépendant, dans son savoir et sa pratique, de l'expérience des 
malades, des visions qu'ils ont de leur mal et du discours collectif sur la maladie. (16)

Les deux semaines d'hospitalisation retranscrites dans Cytomégalovirus confirment malheureusement la solide subsistance de l"'ancien régime". Guibert témoigne de "la patience du patient" requise pour "survivre" dans ce sanctuaire paradigmatique du pouvoir institutionnalisé où règnent en maîtres, médecins et personnel infirmier avec leurs routines administratives et personnelles. ${ }^{13}$ Le style et la présentation dépouillés ainsi que les anecdotes tragi-comiques comme les demandes répétées, jour après jour, d'un obturateur ou d'un pied mobile à perfusion, confèrent au journal une qualité cauchemardesque quasi beckettienne. Après trois jours d'hospitalisation, les micro-exactions constamment subies contraignent Guibert à cette réflexion terrifiante: "Il faut tout de suite se faire respecter, c'est épuisant, un rapport de forces qui dure un ou plusieurs nuits et jours. Ils veulent qu'on perde, ils comptent sur l'usure. Ensuite, selon les cas, ils respectent ou ils laminent" (29). On ne sait selon les remarques de Guibert si la maladie n'est pas moins cause d'épuisement que l'affrontement sans répit qui l'oppose au personnel médical: "Vidé ce soir, comme si j'avais boxé, malade, pendant deux jours et deux nuits, contre une cinquantaine de personnes en principe en bonne santé" (43-44). La fatigue et la perte d'énergie, le prix à payer, ne dissuadent pas Guibert de la pratique de la rébellion douce. Dans un conflit qui l'engage à une infirmière, la confrontation s'avère payante puisqu'elle aboutit en un changement favorable de personnel (41-42). Guibert se fera assurément respecter lorsqu'il refusera de se laisser emmener nu sous la "blouse de papier bleu transparent" de circonstance à travers les couloirs pour une intervention chirurgicale (58). Le service entier sera stupéfait de voir ce patient arriver dans le bloc opératoire en "costume de ville, [s]on chapeau sur la tête et la blouse transparente bleue sur l'épaule" et demander à se changer dans les toilettes (6061). Guibert confirme à travers ce geste excentrique que le pouvoir, s'il fallait encore s'en convaincre, se négocie perpétuellement dans la dynamique que créent formes de résistance et impositions disciplinaires.

Au terme de ce rapide survol de la psychologie et des rapports de pouvoir entre soignants et soignés dans l'institution médicale, subsiste néanmoins une question: pourquoi Guibert se laisse-t-il tenter par des voies alternatives auxquelles, au fond, il ne croit pas? L'ambivalence apparente de Guibert dans ses démarches hétérodoxes ne fait que révéler la douloureuse contradiction entre d'une part l'impotence de la médecine avec sa promesse de guérison déçue, et d'autre part le désir de guérison du malade. Son voyage au Maroc, ainsi que les nombreuses aventures qui le mèneront à travers le monde dans un état de santé déplorable, semblent être des choix faits à la limite de la rationalité face à la certitude d'une mort proche. Mais pour Guibert, c'est une manifestation d'un espoir ultime, existentiel, un espoir "fou". Il s'agit en effet d'une expression de la vie, 
de la "Vraie Vie"-selon l'expression de Pascal de Duve, autre écrivain sideen-, qui cherche à dépasser les limites de contrôle et de prédictions établies par l'institution médicale, et plus généralement à échapper aux contingences et aux conventions de la vie réglée. C'est pourquoi, comme le dit Guibert, les sidéens aiment voyager en dépit des interdictions prononcées par les médecins pour ensuite blâmer ceux-ci si quelque "accident" a lieu (Le Protocole compassionnel 91). Pour Guibert, le voyage et la scriptualisation de la maladie-le "vaccin littéraire"-constituent les deux moyens principaux de réinvention de soimême dans le rapport de forces que la maladie lui impose. La maladie signifie au sidéen une injonction; elle l'assujettit et le constitue. La seule issue envisageable est de créer un nouveau rapport avec la maladie qui ne peut relever de la dérobade ou de l'esquive. Ce nouveau mode d'existence résulte d'une penséeprendre la maladie avec philosophie-qui consiste à infléchir le pouvoir que la maladie peut exercer sur l'individu. Guibert invente ce que Foucault appelle un "processus de subjectivation," une nouvelle éthique ou un art de vivre (Foucault 32-39). ${ }^{14}$ Dans L'Homme au chapeau rouge, dernier roman de la trilogie, le narrateur Guibert se transforme en marchand de tableaux - collectionneur, et développe une nouvelle forme de subjectivité où le monde médical et la maladie apparaissent à peine. Après la mort de Guibert paraît Le Paradis, vague souvenir recomposé d'un narrateur métamorphique dont les fragments de mémoire s'effacent comme par enchantement. Ce narrateur, à la fois Hervé Guibert, H. G. et héritier fictif d'un richissime homme d'affaires, réduit le sida à une trace: élément à la fois central et invisible, une réduction homéopathique dans un monde en train de se dissoudre. Il déclare en parlant de lui-même et de sa partenaire Jayne Heinz: "Nous avons fait le test ensemble, nous n'avons pas le sida" (130). Par ailleurs, dans ce même registre de l'oblitération du monde réel, relevons succinctement que Jayne Heinz est un "non-être", absente des registres d'état civil, qui écoute une non-musique sur des disques compact vierges et lit des livres noncoupés. Le narrateur quant à lui, dans son aspiration à une nouvelle subjectivité aspire à une atomisation de son moi:

Je suis un être double, écrivain parfois, rien d'autre les autres fois, je voudrais être un être triple, quadruple, un danseur, un gangster, un funambule, un peintre, un skieur. J'aimerais faire du delta-plane et me jeter dans le vide, foncer comme un bolide sur des pistes dont la neige serait de l'hérö̈ne. (117)

A la schizophrénisation du sujet s'ajoute une "schizophrénie temporelle" de la narration composée en discontinu de fragments de presents perpétuels avec à l'occasion un ancrage dans le passé mais dont aucun continuum linéaire ne peut être déduit. En somme, le procès d'esquive de la maladie au seuil de la mort passe par une déréalisation du monde référentiel doublée d'une narration fragmentée qui la renforce.

Le voyage et l'écriture sont une tentative de "dépassement" de la ligne 
imposée par le sida ou plus exactement une courbure ou un chevauchement comme le précise Deleuze: "Ployer la ligne pour arriver à vivre sur elle, avec elle: affaire de vie ou de mort" (151). Rappelons que Guibert avait bénéficié d'un "sursis" grâce à la combinaison de DDI et de Prozac, qui l'autorisait à reprendre ses activités de voyage et d'écriture.

C'est le DDI du danseur mort, avec le Prozac, qui écrit mon livre à ma place. Ce sont ces 335 milligrammes de poudre blanche fabriquée à Ickenham, Middlesex, en Angleterre, et cette gélule quotidienne de 20 milligrammes de Fluoxétine Chlorohydrate qui me redonnent la force de vivre, d'espérer; de bander, de bander pour la vie, et d'écrire. (Le Protocole compassionnel 84)

Cette écriture sous influence que Guibert doit au pharmakon chimique repose sur l'adroit maniement d'un double paradoxe. D'abord, l'impossible projet d'écrire sa maladie afin de s'y soustraire, afin d'échapper à son emprise. La double injonction d'écrire et d'oublier sa maladie simultanément est déjouée par une croyance ferme à la magie des mots qui incite Guibert à compromettre sa vie, deuxième paradoxe, par le projet même qui vise à la sauver: "Oui, je peux l'écrire, et c'est sans doute cela ma folie, je tiens à mon livre plus qu'à ma vie; je ne renoncerais pas à mon livre pour conserver ma vie, voilà ce qui sera le plus difficile à faire croire et comprendre" (A l'ami 257). Ce pharmakon littéraire, car le projet d'écriture retient toujours le même potentiel de guérison et de santé, permet à Guibert de se "libérer" momentanément du sida par la juxtaposition de ces deux paradoxes qui, tels des contraires, $s$ 'annulent mutuellement. L'élision de la maladie est encore et toujours fonction d'une dérobade. Le "vaccin littéraire" existe, Guibert l'a inventé.

The Ohio State University 


\section{Notes}

${ }^{1}$ La physiopathologie est l'etude des troubles qui apparaissent dans le fonctionnement des organes au cours d'une maladie.

2 L'apparition et le succès populaire de la plupart des médecines parallèles, toujours en vogue à l'heure actuelle, concordent avec le développement de la médecine moderne durant la deuxième moitié du dix-neuvième siècle. Le chapitre "Histoire de la médecine" par Pierre Huard et Marie-José Imbault-Huart de l'encyclopédie de la Pléiade Médecine (Vol.1) mentionne la "Christian Science," l'osthéopathie, la chiropraxie, les régimes végétariens, le naturisme, l'énorme succès en fin de siècle de différentes écoles d'hypnose, ainsi que le développement de l'homéopathie et de l'acupuncture (614-16).

3 L'urographie est un examen médical qui consiste à radiographier l'appareil urinaire à l'aide d'une substance opaque aux rayons $\mathrm{X}$, injectée par voie intraveineuse.

${ }^{4} \mathrm{La}$ fibroscopie est un examen médical, douloureux et délicat, qui consiste à ausculter par voie orale l'appareil digestif et à effectuer si nécessaire un prélèvement de tissus des parois de l'oesophage et de l'estomac généralement.

5 La coloscopie est un examen endoscopique du colon. Tous les examens mentionnés dans ce passage par Guibert sont pratique courante dans le traitement de diverses maladies opportunistes associées au sida.

${ }^{6}$ Le sida n'est à vrai dire pas une maladie mais un état d'immuno-déficience qui laisse à une multitude d'affections_-souvent rares—la possibilité de se développer. A défaut de cure et outre les médicaments tels que AZT et DDI qui dans la plupart des cas ralentissent le développement du virus, la stratégie jusqu'à aujourd'hui est de soigner les affections lorsqu'elles apparaissent. Un travail de prévention s'effectue également sur les maladies les plus opportunistes.

${ }^{7}$ C'est manifestement le cas en cette fin de siècle avec le sida comme ce le fut au siècle passé avec la syphilis et la tuberculose.

8 La crudothérapie, connue aussi sous le nom d'instinctothérapie, est une méthode therapeutique alternative qui consiste à ne consommer que des aliments crus et frais, sous leur forme naturelle, sans transformation, ni mélange. La méthode a pour but de permettre à l'adepte de retrouver un instinct alimentaire inné qui lui indiquerait l'aliment, selon l'odeur et le goût, dont le corps a besoin. Cette pratique repose sur le principe que l'alimentation (non-naturelle) est la cause principale des maladies.

${ }^{9} \mathrm{La}$ "méthode Coué" est une technique de psychothérapie qui utilise l'hypnose et notamment l'autosuggestion comme moyen de guerison.

${ }^{10}$ Michel Foucault développe la thématique de contrôle, de régulation 
et de résistance en particulier dans Surveiller et punir: Naissance de la prison (Paris: Gallimard,1975) et La Volonté de savoir (Paris: Gallimard, 1976). Il tient pour principe explicatif que le pouvoir se définit dans une dynamique perpétuelle entre opérations régulatoires exercées par les structures hégémoniques et la résistance qu'elles occasionnent.

11 Voir à ce sujet l'excellent ouvrage de deux sociologues Claudine Herzlich et Janine Pierret Malades d'hier, malades d'aujourd'hui (Paris: Payot, 1984).

12 On observe depuis une dizaine d'années le retour en force de maladies comme la tuberculose, la peste et les maladies vénériennes en général. Les causes généralement admises sont autant d'ordre biologique que social. L'usage irraisonné des antibiotiques (qui ont provoqué des mutations inattendues) et les changements de mode de vie (voyages rapides à travers le monde, urbanisation, dégradation de l'aide sociale et médicale...) sont les causes le plus souvent citées.

13 Linda Singer, dans Erotic Welfare (New York: Routledge, 1993), relate l'expérience de sa propre hospitalisation et présente ses réflexions sur le fonctionnement des hôpitaux qui rejoignent à bien des égards celles d'Hervé Guibert. Voir en particulier le chapitre "Hospitalization and AIDS".

14 Michel Foucault utilise le terme de "subjectivation" pour la première fois lors de sa dernière interview réalisée par André Scala et Gilles Barbedette, et publiée dans Les Nouvelles du 28 juin 1984. La traduction anglaise par Thomas Levin et Isabelle Lorenz paraît dans le numéro de l'été 1985 de Raritan, et est reprise dans Michel Foucault - Politics, Philosophy, Culture. Interviews and Other Writings 1977-1984. Ed. Lawrence D. Kritzman.. 


\section{Ouvrages cités}

Apter, Emily. "Fantom Images: Hervé Guibert and the Writing of 'sida' in France." Writing AIDS - Gay Literature, Language, and Analysis. Eds. Timothy F. Murphy \& Suzanne Poirier. New York: Columbia UP, 1993.

Deleuze, Gilles. Pourparlers. Paris: Minuit, 1990.

Duve, Pascal de. Cargo vie. Paris: Lattès, 1993.

Foucault, Michel. L'Usage des plaisirs. Paris: Gallimard, 1984.

Glucksmann, André. La Fêlure du monde. Paris: Flammarion, 1994.

Guibert, Hervé. A l'ami qui ne m'a pas sauvé la vie. Paris: Gallimard, 1990.

---. Le Protocole compassionnel. Paris: Gallimard, 1991.

-.-. Cytomégalovirus. Paris: Seuil, 1992.

---. L'Homme au chapeau rouge. Paris: Gallimard, 1992.

--.. Le Paradis. Paris: Gallimard, 1992.

Herzlich, Claudine et Janine Pierret. Malades d'hier, malades d'aujourd'hui. Paris: Payot, 1984.

-Illich, Ivan. Némésis médicale. Paris: Seuil, 1975.

Huart Pierre, et Marie-José Imbault-Huart. "Histoire de la médecine." Médecine. (vol. 1) Paris: Gallimard, 1980. Sous la direction de Pierre de Graciansky et Henri Péquignot. 2 vols.

Kritzman, Lawrence D., ed. Michel Foucault - Politics, Philosophy, Culture. Interviews and Other Writings 1977-1984. New York: Routledge, 1988.

Médecine. Encyclopédie de la Pléiade (Vol.1). Sous la direction de Pierre de Graciansky et Henri Péquignot. Paris: Gallimard, 1980.

Singer, Linda. Erotic Welfare. New York: Routledge, 1993.

Soljenitsyne, Alexandre. Le Pavillon des cancéreux. Paris: Julliard, 1968.

Tissot, René. "Psychologie médicale." Médecine. (vol. 1) Paris: Gallimard, 1980. Sous la direction de Pierre de Graciansky et Henri Pequignot. 2 vols. 

